\title{
A four-step model for integrating pedagogy to technology in fostering students' meaningful learning
}

\author{
Thwin, E.P.A

\section{Introduction} \\ The COVID-19 pandemic has sped up the use \\ of technology in medical education, and \\ technology becomes an essential educational \\ tool for designing teaching and learning \\ activities. However, selection and how best to \\ use technology can be challenging for \\ educators, especially those new to online \\ The TLAs should achieve the ILOs defined in \\ step 1, encourage students' active participation, \\ create opportunities for them to work in a group, \\ facilitate their reflective thinking, and help them \\ apply their knowledge and skills to real-world \\ contexts. When students engage in intentional, \\ active, collaborative, reflective and authentic \\ learning activities, their learning is meaningful \\ (Howland et al., 2011).
} teaching.

This short article presents a simple four-step model to select and integrate technology into teaching and learning practices. A faculty development workshop on designing online activities I attended in 2016 inspired me to develop the model.

\section{Step 1: Defining the intended learning outcomes}

The first step of the model is to define the intended learning outcomes (ILOs). The ILOs specify the expected levels of students' understanding and performance, and educators can use the Structure of the Observed Learning Outcomes (SOLO) taxonomy (Biggs and Tang, 2015) or the Revised Bloom's taxonomy (Krathwohl, 2002) for defining the ILOs.

\section{Step 2: Designing teaching and learning activities}

The second step is to design the teaching and learning activities (TLAs).

Department of Medical Sciences and Paramedicine, School of Health and Social Sciences, Nanyang Polytechnic, Singapore.

Corresponding author: Dr Eugenie Phyu Aye Thwin eugenie_phyu_aye_thwin@nyp.edu.sg

\section{Step 3: Exploring the potential technological} tools

The third step is to explore the potential technological tools to support the TLAs designed in step 2. As various technological tools are available nowadays, selecting suitable tools for the TLAs can pose a challenge to educators. Therefore, they should start with simple and easy-to-use tools and seek technical support from their schools if needed. Using too many tools can frustrate both students and educators as they need to log in to different platforms, remember several passwords and familiarise themselves with additional features; thus, educators should limit the number of tools used in a classroom (The University of New South Wales, 2019).

Step 4: Mapping the TLAs from step 2 to the tools explored in step 3

The last step is to map the TLAs and potential tools by constructing a matrix table. The table should include four columns representing the four steps, and the rows should appoint the ILOs of a lesson. Using the matrix table, educators can visualise the constructive alignment of ILOs, TLAs and technological tools. 
After introducing the four-step model, I select a topic from the cardiovascular system (Martini and Bartholomew, 2016) to illustrate the mapping of pedagogy to technology. The first two levels of cognitive domains from Revised Bloom's taxonomy (Krathwohl, 2002) are used to define the ILOs.

Example (1)

Step 1: The first level of the cognitive domain of Revised Bloom's taxonomy (remembering) is used to describe the ILO for the first example. I define the ILO as "students should be able to list the major arteries and veins of the pulmonary circulation".

Step 2: We can design the TLAs for this ILO as self-assessment quizzes. Activities such as drag and drop or multiple-choice questions with immediate feedback will encourage students to engage actively.

Step 3: The potential technological tools for quizzes can be Socrative, Mentimeter, Kahoot, or the Blackboard quizzes.

Step 4: Among the tools identified in step 3, I choose Mentimeter and the Blackboard quizzes because these tools are user-friendly and have institutional licenses.

\section{Example (2)}

Step 1: The second level of the cognitive domain of Revised Bloom's taxonomy (understanding) is used to define the ILO for the second example. I describe the ILO as "students should be able to explain the cardiovascular homeostatic responses to exercise".

Step 2: We can design the TLAs for this ILO as a group discussion where students engage in dialogue with their peers and explain their understandings of the ILO to fellow students.

Step 3: The potential collaborative tools to support the TLAs can be Padlet, Google Docs, or Discussion Forum in the Blackboard. The discussion can be synchronous or asynchronous, and students can upload pictures, images, videos, or other multimedia materials to make their discussions lively and engaging.
Step 4: Among the tools identified in step 3, I select Padlet and the Blackboard Discussion Forum because they are easy to use and have institutional subscriptions.

\section{Conclusion}

As conventional chalk-and-blackboard teaching has migrated to technology-integrated teaching, informed decisions on selecting technological tools can be critical for facilitating students' learning. I hope this article may help educators align the ILOs, TLAs and technologies and use technologies as engagers, facilitators, supporters, collaborators, and intellectual partners to foster students' learning.

\section{Acknowledgement}

I wish to acknowledge Dr Pratima Majal, Assistant Director, Learning Academy, Temasek Polytechnic, Singapore, for introducing me to the concept of meaningful learning and designing online activities.

\section{Statement of Conflicting Interest}

The author has no conflicts of interest to declare.

\section{References}

Biggs, J. \& Tang, C. 2015. Constructive Alignment: An Outcomes-based Approach to Teaching Anatomy. In: CHAN, L. K., \& PAWLINA, W., (ed.) Teaching Anatomy A Practical Guide. 1st ed.: Springer.

Howland, J. L., Jonassen, D. H. \& Marra, R. M. 2011. Goal of Technology Integration: Meaningful Learning. Meaningful Learning with Technology. 4th ed.: Pearson.Krathwohl, D. R. 2002. A Revision of Bloom's Taxonomy: An Overview. Theory Into Practice, 41, 212-218.

Martini, F. H. \& Bartholomew, E. F. 2016. The Cardiovascular System: Blood Vessels and Circulation. Essentials of Anatomy \& Physiology. 7th ed.: Pearson Education.

The University Of New South Wales. 2019. Selecting Technologies [Online]. Available at: https://teaching.unsw.edu.au/selectingtechnologies?fbclid=IwAR39HfgP7cBJ1p9jBloJdtwXW17E2kqeQn647PAScrSnKzZ-71HOmcKsk [Accessed 04/07/2021]. 